\title{
Power Grid Operation and Maintenance System Based On LBS
}

\author{
Fei Chen ${ }^{1+}$ \\ ${ }^{1}$ Information Center, Guangdong Power Grid Corporation \\ ${ }^{1}$ No. 836 of Dongfengdong Road, Yuexiu District, Guangzhou, Guangdong Province, China
}

\begin{abstract}
This paper describes a LBS-based operation and maintenance system which contains two parts, Webserver and client. The client part is based on Android and the Server is based on Django framework in Python. The whole system is based on Http protocol and implement the acquisition route, route planning, navigation.
\end{abstract}

Keywords: Android, Django, Navigation.

\section{Introduction}

With the surge of electricity consumption in the industry, commerce, agriculture and the daily life, the good working performance of power equipment is more and more important, therefore the cost of on-site maintenance is becoming higher and higher. In the rural areas with deficient geographic reference information, there is no buildings and routes available for reference in the existing navigation software, and some places even have no signal of satellite map. So, in the traditional way of power equipment management and maintenance, the maintainer may need to ask the villagers to find a destination and write down some records about the equipment; the next time, when a new collector or maintenance staff to work, he still need to spend a lot of time to locate the household. This will undoubtedly increase the burden on the grid staff, resulting in low efficiency. So how to provide personalized location and route service for the maintainer of rural power grid? How to use the smart phone platform to promote the informatization of power industry? Those are worth thinking.

The power grid operation and maintenance system based on LBS is mainly for maintenance personnel. According to the practical needs of the specific work scenarios, we divide the resident role into two parts: The first part is the data collector, mainly responsible for rural power equipment information collection and upload, including the location data of electricity meter and the routes connecting them; The second part is the maintenance personnel, they download the latest route data and use them to go to the target place, repair the meter and update its status records. All the operators can use the route planning function to query the existing shortest path between different households, as well as adding notes in a variety of ways.

\section{The principle of Power grid operation and maintenance system}

On the whole, power grid operation and maintenance system consists of two parts: cloud server and Android ${ }^{[1]}$ client;

- the client connects to the cloud server via Internet;

- the system uses the HTTP protocol to realize the communication between the client and the cloud server, including the synchronization of the location data, the route data and the resident data between client and server;

System deployment:

+ Corresponding author. Tel.: +8618802018822.

E-mail address: rhine.chan@foxmail.com 
- A, server database: Linux, MySQL

- B, Web applications: Linux, Django

- C, client: Android ${ }^{[2]}$ App, SQLite, AMap map SDK

\section{Software Design for Server}

\subsection{The software diagram for server}

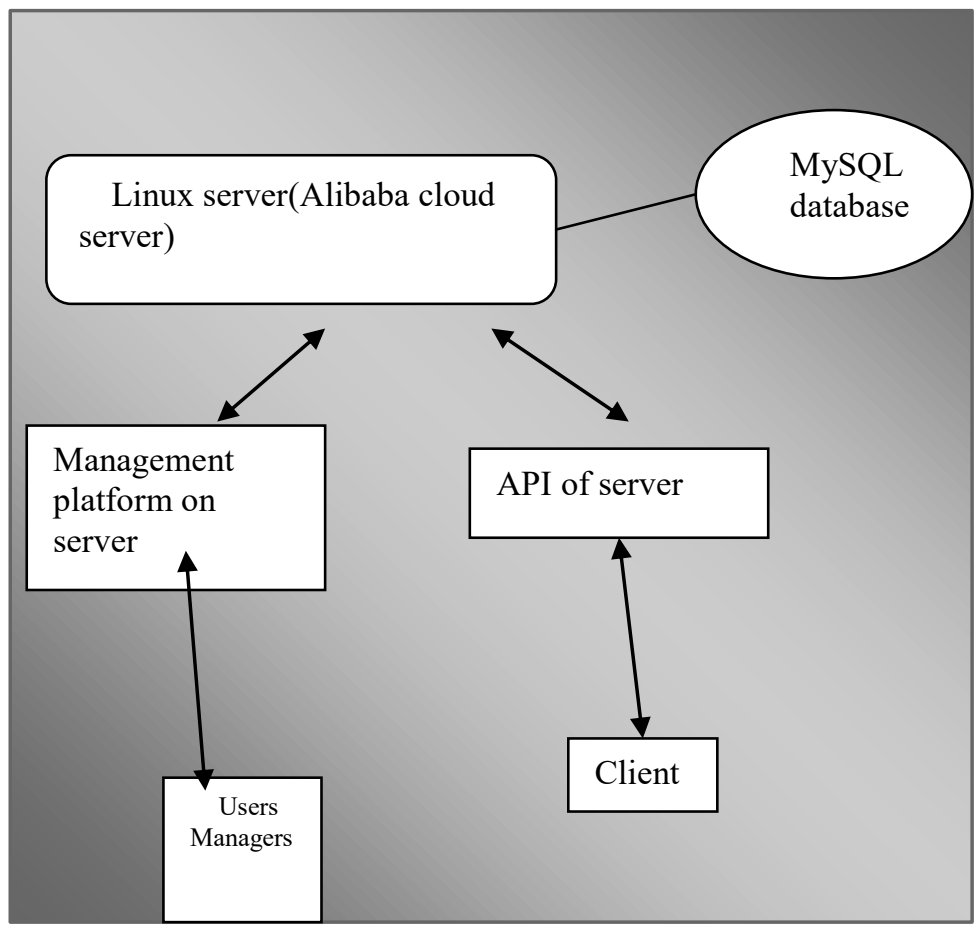

Fig. 1: software structure diagram of server.

The server uses Python based Django ${ }^{[3]}$ framework, and adopts the request-response mode of HTTP to implement the server interfaces and functions. The client send a request according to the concrete function logic to the specific server interface, then the server receives the request and process the data. After processing, the server will return a response code and some related data as a result to the client.

\subsection{API workflow on Server}

The Android client can be used to collect routes and resident information, and upload new data to server through the network. The server will process the request, accept data and save to the MySQL database. Similarly, when get a download request from a client, the server will reads specific data for database and send them in agreed format. All data are transmitted in JSON format.

The main interactive interfaces for client and server:

- The client can access to the latest routes information.

- The client can access to resident information.

- The client can upload the newly collected routes information to the server.

- The client can update the resident data to the server.

\subsection{Management platform on Server}

In order to facilitate the staff and managers to manage and query the data on the cloud server, the system designed and implemented a web platform which based on Django ${ }^{[3]}$ templates, presenting the content in the form of web pages.

The platform mainly achieved the following function:

- Import and Export of resident information: the managers can add or export lot size of resident information by operating the Excel table. 
- Import and Export of resident's location information: the managers can add or export lot size of resident's latitude and longitude information by operating the Excel table.

- Add, Delete, Check and Change any data in the cloud database. (The system will record the actions.)

- Manage system user accounts and permissions, add new administrator, modify passwords, etc.

\section{Client Software Design}

\subsection{Client software architecture}

Power grid operations system client is based on the Android platform. According to the actual work needs of power grid staff, the system is designed of the five functional modules: path acquisition, general operations, user information modification, data updates, and offline map management. Path acquisition and general operations based on LBS services, using the Auto Navigation Software Co application SDK on developer platform to develop the main function. The module for the business logic is shown below:

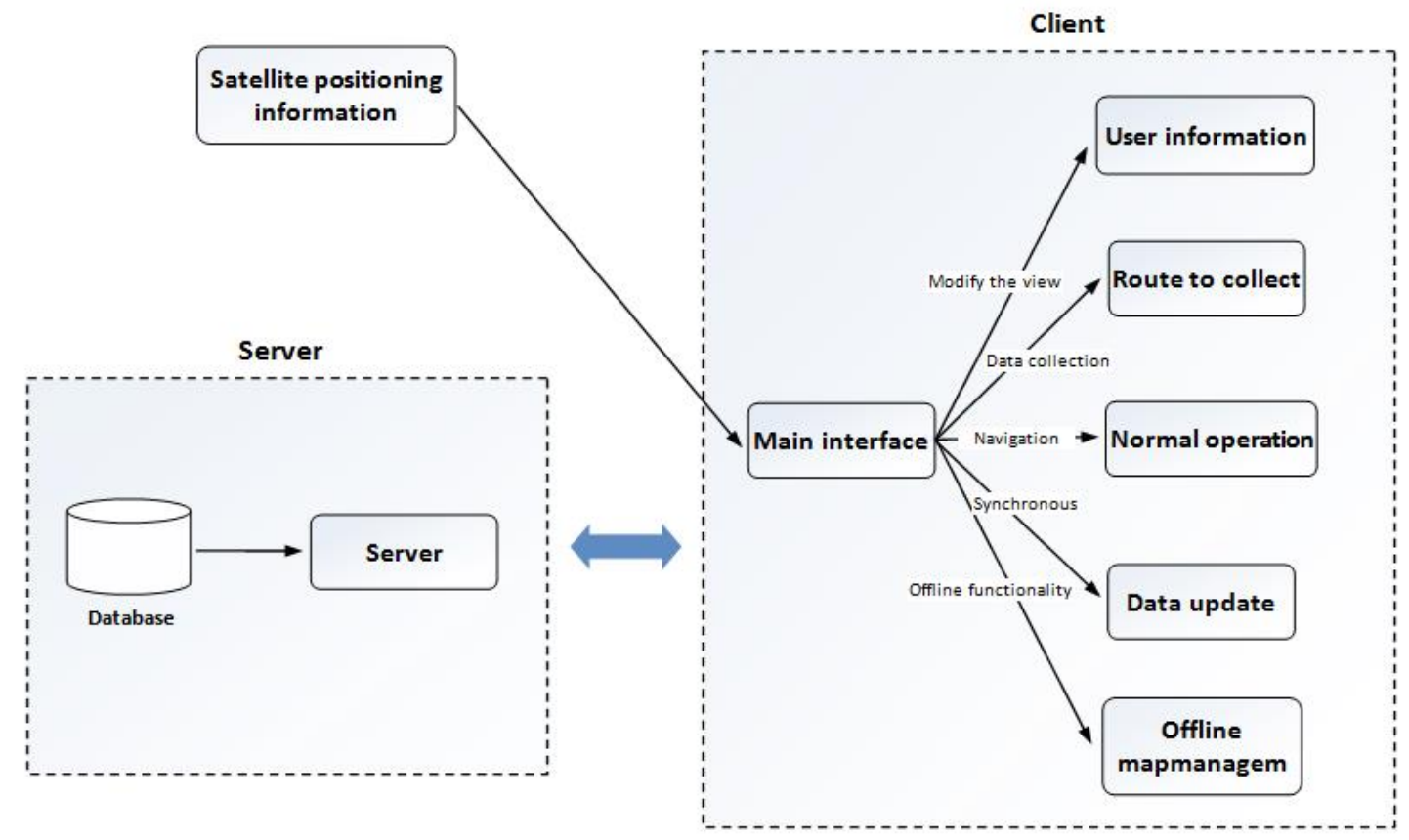

Fig. 2: software architecture of client

\subsection{Client database design}

The client SQLite database includes the following four tables

Users table: Including the user name, telephone, address, meter bar code, latitude and longitude and other 17 fields

Route table: Including path ID, starting point, end point, longitude, latitude, collectors and so on

Node table: The start / end of each route in the Route table is the node on the road (via point), the logging node facilitates the path query

Location table: Record all coordinate points that appear on each path in the Route table in the order of positioning, including the node

\subsection{Module design of client business logic}

(1) Path acquisition module

The module consists of three pages: positioning and path drawing page, via point (node) information record page and location display page. Positioning and path drawing page contains three buttons and a map, three buttons function is basically the same. By clicking the button to enter the information page. Based on the software user job flow is divided into "start", "add point", "end".

Open the software in the main page and click on the "path acquisition" will enter the path drawing page and initialize the location of resources and start positioning. 


\section{(2)General operations module}

General operations module includes two functions: path planning and navigation.

In the process of path acquisition process, every recorded point can be queryed as a starting point or end point. The user ID, user name or other location point part of the name can be the part of query. Click the input box on the right of the search icon main to fuzzy query the Users table and the Node table. The query results are presented in a list, the list entry will show similar user ID number, account name, address or other location point name, the area name, etc. The query according to more detailed information to select the appropriate location.

After selecting the location point in the Node table, the corresponding path in the Node table and the corresponding path in the Route table can constitute the undirected graph, and then use the shortest path algorithm SPFA to calculate the shortest path between a fixed start and end point, The AutoNavi API draws the segment on the map.

Real-time navigation, that is, draw a dotted line between the actual location of the operator and the query starting point, indicating the operator generally forward direction. This part of the interface can be achieved with the AutoNavi API interface and mapping interface.

(3)User information modification module

The user information modification part has been integrated in the way through the point of the record page. Check the electricity account information according to the account number, account name, table and the terminal number and other inquiries, use the positioning function to modify the latitude and the electricity address of the user and pay attention to the status of electrical equipment maintenance status. This part of the function only involves the users table query and update

(4)Other modules

The data update module includes the path information upload, the download, the user information modification upload, the download. For the database, involving all the tables of the query, update, insert and data communication between the server and interpretation, mainly used Http request, JSON ${ }^{[4]}$ package analysis technology。

Offline map management is to facilitate the smooth display of specific area map data without network conditions, so as to ensure that some business functions are not restricted by the network。

\section{The Ending}

This paper designed and implemented a power grid operation and maintenance system which based on Android LBS and Django server. With the help of AMap SDK, GPS and other mature tools, by some simple operation, the client can realize the information collection of routes and residents, as well as the navigation based on the self-collected data. Because of the powerful computing and storage capabilities of cloud server, and the convenient management of web pages, the system is very simple and convenient.

This design idea can be widely used in practical fields that need GPS navigation, route planning and other map functions. It has a wide promotional significance and the potential value of the business in the era of mobile Internet and mobile office.

\section{References}

[1] Yan Wei, Ye Jiankao, Application of multithreading technology in Android mobile development[J], Information and communication, 2012.

[2] Yan Junxia, Zhou Yan. Development of Mobile based on Google Android platform[J]; Silicon Valley, 2012.

[3] Xue Yaowei,Management interface module is automatically generated basedon the Django framework design and implementation, Anhui University, 2013.

[4] Li Xin,Research and implementation of information interactive system based on JSON, University of posts and telecommunications, 2006. 